\title{
Efficient Execution Plans for Distributed Skyline Query Processing
}

\author{
João B. Rocha-Junior,' Akrivi Vlachou, Christos Doulkeridis, and Kjetil Nørvåg \\ Department of Computer and Information Science \\ Norwegian University of Science and Technology (NTNU), Trondheim, Norway \\ \{joao,vlachou,cdoulk,noervaag\}@idi.ntnu.no
}

\begin{abstract}
In this paper, we study the generation of efficient execution plans for skyline query processing in large-scale distributed environments. In such a setting, each server stores autonomously a fraction of the data, thus all servers need to process the skyline query. An execution plan defines the order in which the individual skyline queries are processed on different servers, and influences the performance of query processing. Querying servers consecutively reduces the amount of transferred data and the number of queried servers, since skyline points obtained by one server prune points in the subsequent servers, but also increases the latency of the system. To address this trade-off, we introduce a novel framework, called SkyPlan, for processing distributed skyline queries that generates execution plans aiming at optimizing the performance of query processing. Thus, we quantify the gain of querying consecutively different servers. Then, execution plans are generated that maximize the overall gain, while also taking into account additional objectives, such as bounding the maximum number of hops required for the query or balancing the load on different servers fairly. Finally, we present an algorithm for distributed processing based on the generated plan that continuously refines the execution plan during in-network processing. Our framework consistently outperforms the state-of-the-art algorithm.
\end{abstract}

\section{Categories and Subject Descriptors}

H.2.4 [Database Management]: Systems-Query processing

\section{General Terms}

Algorithms, Experimentation, Performance

\section{Keywords}

Skyline query, distributed systems, execution plan

*On leave from the Universidade Estadual de Feira de Santana.

Permission to make digital or hard copies of all or part of this work for personal or classroom use is granted without fee provided that copies are not made or distributed for profit or commercial advantage and that copies bear this notice and the full citation on the first page. To copy otherwise, to republish, to post on servers or to redistribute to lists, requires prior specific permission and/or a fee.

EDBT 2011, March 22-24, 2011, Uppsala, Sweden.

Copyright 2011 ACM 978-1-4503-0528-0/11/0003 ...\$10.00

\section{INTRODUCTION}

The importance of skyline queries [4] has been recognized for applications that involve decision-making. Skyline queries do not require an explicit preference function, which may be difficult for the user to define when the relative importance of the different criteria is vague. Consider for example a real-estate database containing information about houses, represented as tuples. Each tuple stores different characteristics of a house, for instance its price and its distance to a point of interest, such as the nearest metro station. A person that is interested in a house that is cheap and close to a metro station poses a skyline query to retrieve the best offers. The houses in the skyline set are those that are not worse than (not dominated by) any other house in both price and distance.

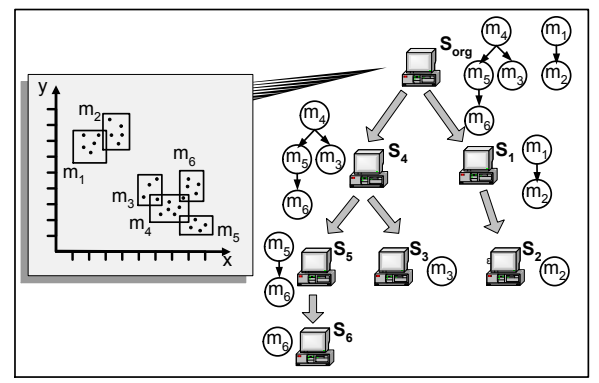

Figure 1: Distributed skyline query processing.

Skyline queries have been studied both in centralized and distributed environments $[3-5,7,10,13,14,16,18,20-24]$. However, planning the execution of skyline queries on different (potentially overlapping) data fractions, in order to obtain the skyline set of the entire dataset efficiently, has not received adequate attention in the related work. This problem is particularly important and challenging in distributed environments, where each server stores only a fraction of the available data. Moreover, with the advent of large-scale data centers and cloud computing infrastructures, data is increasingly stored and processed in a distributed way. Thus, it is necessary to efficiently support complex query types, such as skyline queries, in distributed environments.

Figure 1 depicts an example of a dataset distributed over multiple servers, $S_{1}$ to $S_{6}$. The query originator $S_{\text {org }}$ can directly communicate with any other server. Each server $S_{i}$ stores autonomously a fraction of the data that in this example correspond to the data points enclosed in the rectangle $m_{i}$. In order to retrieve the skyline set of the distributed 
dataset, all servers compute the skyline set of the locally stored data points. Then, $S_{\text {org }}$ gathers these points and computes the skyline set of the entire dataset. Before processing the distributed query, $S_{\text {org }}$ first derives an execution plan that defines the order in which the query is processed on the different servers.

The execution plan influences the overall performance of the query. For example, depending on the execution plan, some servers do not have to be contacted at all. In Figure 1, $S_{6}$ can be removed from the execution plan after processing the skyline query at $S_{4}$, since there exists a point stored locally at $S_{4}$ that dominates all points stored at $S_{6}$. Furthermore, the number of data points transferred back to $S_{\text {org }}$ can be drastically reduced, by discarding data points stored at $S_{i}$ that are dominated by skyline points of servers that precede $S_{i}$ in the execution plan. In addition, the efficiency of local query processing at $S_{i}$ can be improved by using the skyline points of previous servers to immediately discard points stored locally at $S_{i}$. On the other hand, the execution of local skyline queries on consecutive servers is a blocking operation and may lead to increased response time. Motivated by this discussion, in this paper, we study the problem of creating efficient execution plans that improve the performance of the distributed skyline query.

Our main observation is that a dependency must exist between consecutive servers, in order to obtain any performance gain by reducing the number of contacted servers or transferred data. A dependency between two servers means that points of the first server must dominate at least some points of the following server. Therefore, our approach optimizes the performance of distributed skyline computation, by exploiting the dependencies - when they exist - between skyline queries on different servers. The dependencies between skyline queries are complex, and their representation forms a directed graph with cycles. For example, if the servers $S_{3}$ and $S_{4}$ are considered, both local skyline queries depend on each other. Thus, it is not feasible to incorporate all dependencies in the execution plan, and the major challenge is to derive an execution plan that includes the most promising dependencies. Our proposed framework, called SkyPlan, quantifies the efficiency of an execution plan based on the potential gain obtained by querying different servers consecutively (henceforth this gain is mentioned as pruning power), and generates cost-aware execution plans. In more details, we make the following contributions:

- We propose SkyPlan, a framework that generates costaware execution plans with maximum pruning power and drastically reduces the response time of distributed skyline query processing.

- We extend our framework to produce multi-objective execution plans, when additional objectives - other than maximizing the pruning power - need to be fulfilled simultaneously.

- We propose an efficient algorithm that exploits the generated execution plan for processing skyline queries in a distributed environment. We further enhance the performance of query execution by continuously refining both the execution plan and the filter points, as the query is being processed in a distributed manner.

- Finally, we perform an extensive experimental evaluation that demonstrates that SkyPlan consistently outperforms the state-of-the-art algorithm [7] by $1-3$ orders of magnitude.

The remaining of this paper is organized as follows: Section 2 overviews the related work. Then, we present the necessary preliminaries in Section 3 and the overview of SkyPlan in Section 4. In Section 5 the dependency graph is defined, while in Section 6 the quality of a cost-aware execution plan is introduced and the plan generation algorithm is presented. In Section 7, we describe multi-objective execution plans that satisfy additional constraints. Section 8 presents the distributed skyline computation guided by the execution plan. Finally, the experimental evaluation is presented in Section 9 and we conclude in Section 10.

\section{RELATED WORK}

Skyline computation [4] has recently attracted considerable attention and has been studied in a variety of distributed systems, including web information systems [3] parallel systems [17], peer-to-peer systems $[5,10,13,18,19$, 21-23], mobile ad-hoc networks [14,20], as well as more generic distributed systems $[7,16,24]$. In the following, we provide an overview of existing approaches for distributed skyline computation. One of the first approaches, by Balke et al. [3], focuses on skyline query processing over multiple sources, with each source storing only a subset of attributes (vertical data distribution). Afterwards, most of the existing approaches focus on highly distributed environments, such as peer-to-peer networks, assuming that all data sources store common attributes (horizontal data distribution). Such approaches can be classified in two categories.

In the first category, the proposed methods assume space partitioning among servers, thus each server is responsible for a disjoint partition of the data space. Several approaches belong to this category, namely DSL [23], SSP [21] SkyFrame [22] and iSky [5]. DSL was proposed by Wu et al. [23] and it is the first paper that addresses constrained skyline query processing over disjoint data partitions by using a structured peer-to-peer overlay, namely CAN. Wang et al. [21] propose the SSP algorithm based on the use of a treebased peer-to-peer overlay (BATON) for assigning data to servers. Later, the authors present SkyFrame [22] as an extension of their work. Chen et al. [5] propose the iSky algorithm, which employs an alternative transformation, namely iMinMax, in order to use the BATON overlay.

In the second category, data partitioning is assumed and each server autonomously stores its own data. Hose et al. [13 use distributed data summaries for efficient processing of approximate skyline queries and provide guarantees for the completeness of the result. Skyline computation over a superpeer architecture has been studied in $[18,19]$. SKYPEER [18] transforms the multi-dimensional data into one-dimensional values and utilizes a thresholding scheme in order to reduce the transferred data. SKYPEER+ [19] extends SKYPEER, by focusing on efficient routing of skyline queries over the super-peer network. Fotiadou et al. [10] propose BITPEER for supporting efficiently continuous subspace skylines in a distributed setting by using distributed bitmap indexes. Huang et al. [14] study skyline query processing over mobile ad-hoc networks. In [20], bandwidth-constrained skyline queries in mobile environments are studied.

In addition, a few methods have been proposed that assume data partitioning among servers without the restriction 
of an existing overlay network, i.e., the query originator can directly communicate with all servers. Cui et al. [7] proposed the PaDSkyline algorithm, where the data stored at each server are summarized by MBRs. Initially, the MBRs of all servers are collected and partitioned to incomparable groups. For each group of MBRs, an intra-group query execution order is defined and the authors propose two strategies. The first is the linear execution of queries on servers, where the MBRs are sorted based on their Euclidean distance to the origin of the data space. The second strategy is to build a tree that defines the execution order, but the generated tree is strongly dependent on the order that the MBRs were collected and processed. Assuming the same architecture, in [16], an approach called AGiDS is proposed that uses a grid-based data summary of the data stored locally at each server for defining the execution order. In [24], a feedback-based distributed skyline (FDS) algorithm is proposed, which aims to minimize the bandwidth consumption at the expense of several round-trips.

Our framework, similarly to $[7,16,24]$, makes no assumption on the existence of a specific overlay network. Differently than [7], we focus on the execution plan which influences the overall performance of the distributed query processing. In contrast, the execution order proposed in [7] does not take into account the gain of querying two servers consecutively and thus, does not optimize the performance of query processing. In addition, we propose a distributed query execution mechanism that dynamically eliminates parts of the plan and constantly refines the filter points. Furthermore, SkyPlan uses MBRs for data summarization in contrast to AGiDS [16] that uses a grid-based data summary. The main shortcoming of AGiDS is that it assumes that the partitions of the grid-based data summary are common and known a-priori to all servers, which is not feasible for large and highly dynamic distributed systems. Moreover, AGiDS does not produce tree-based execution plans, in contrast to SkyPlan. Finally, SkyPlan focuses on minimizing the response time, which is different than the goal of FDS [24], since FDS requires several round-trips to process the query, thus it incurs high response time.

\section{PRELIMINARIES}

Given a dataset $D$ on a data space defined by a set of $d$ dimensions $\left\{d_{1}, \ldots, d_{d}\right\}$, a point $p \in D$ is represented as $p=\left\{p_{1}, \ldots, p_{d}\right\}$ where $p_{i}$ is the value on dimension $d_{i}$. Without loss of generality, we assume that $\forall d_{i}: p_{i} \geq 0$, and that smaller values are preferable.

Definition 1. A point $p \in D$ dominates another point $q \in D$, denoted as $p \prec q$, if (1) on every dimension $d_{i}$, $p_{i} \leq q_{i}$; and (2) on at least one dimension $d_{j}, p_{j}<q_{j}$. The skyline is a set of points $S K Y$ which are not dominated by any other point in $D$.

Consider the example in Figure 2(a), where each point represents a hotel and the $y$-dimension represents the price of a room, while the $x$-dimension captures the distance of the hotel to the beach. A hotel dominates another hotel because it is cheaper and closer to the beach. Thus, the skyline points $(a, i, m$ and $k$ ) are the best possible tradeoffs between price and distance from the beach.

The notion of skyline queries can be extended to constrained skyline queries. Given a set of constraints, a con-
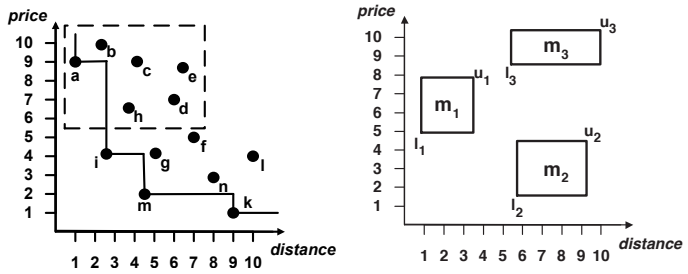

(a) Skyline example.

(b) Domination example.

Figure 2: Skyline and domination examples.

strained skyline query returns the skyline points by considering only the data points enclosed in the data space defined by the constraints. Each constraint is expressed as a range along a dimension and the conjunction of all constraints forms a hyper-rectangle in the $d$-dimensional data space. For example, the dashed rectangle in Figure 2(a) defines a constrained skyline query and the constrained skyline points are $a$ and $h$. A hyper-rectangle $m_{i}\left(l_{i}, u_{i}\right)$ is represented by two points, its lower left corner $l_{i}$ and its upper right corner $u_{i}$. The dominance relationships between two hyper-rectangles $m_{i}$ and $m_{j}$ are described as follows:

DeFinition 2. Given two hyper-rectangles $m_{i}$ and $m_{j}$, the dominance relationships are: (1) $m_{i}$ dominates $m_{j}$, if $u_{i} \prec l_{j}$; (2) $m_{i}$ partially dominates $m_{j}$, if $l_{i} \prec u_{j}$, but $u_{i} \nprec l_{j}$; (3) $m_{i}$ and $m_{j}$ are incomparable, if $l_{i} \nprec u_{j}$ and $l_{j} \nprec u_{i}$.

For example in Figure 2(b), $m_{1}$ and $m_{2}$ are incomparable, which means that no point enclosed in $m_{1}$ can dominate any point in $m_{2}$ and vice versa. Moreover, $m_{1}$ dominates $m_{3}$ (since $u_{1}$ dominates $l_{3}$ ), while $m_{2}$ partially dominates $m_{3}$ (since $l_{2}$ dominates $u_{3}$ but $u_{2}$ does not dominate $l_{3}$ ). We also mention that a point $p$ dominates a hyper-rectangle $m_{i}\left(l_{i}, u_{i}\right)$ if and only if $p \prec l_{i}$, i.e., point $p$ dominates the lower left corner of $m_{i}$.

As stated in [7], the volume of the dominance area quantifies the pruning power of a data point. The dominance area of a point $p$ is defined as the area that is enclosed by the hyper-rectangle that has as lower left corner the point $p$ and as upper right corner the maximum corner of the universe. Furthermore, given a hyper-rectangle $m_{i}$, we introduce the notion of enclosed dominance area of a point $p$, as the area within the hyper-rectangle $m_{i}$ that is dominated by $p$. Similarly, given two hyper-rectangles $m_{i}$ and $m_{j}$, the enclosed dominance area $V_{i j}$ of $m_{i}$ on $m_{j}$ is the volume of $m_{j}$ that is dominated by the lower left corner $l_{i}$ of $m_{i}$. Based on the above notions, we can define the pruning power $P P_{i j}$ of $m_{i}$ on $m_{j}$.

Definition 3. Given two hyper-rectangles $m_{i}$ and $m_{j}$, the pruning power $P P_{i j}$ of $m_{i}$ on $m_{j}$ is defined as $P P_{i j}=$ $\frac{V_{i j}}{V_{j}}$, where $V_{j}$ denotes the volume of $m_{j}$ and $V_{i j}$ denotes the enclosed dominance area of $m_{i}$ on $m_{j}$.

\section{SKYPLAN FRAMEWORK}

In our system model, a set of $|S|$ servers $S_{i}$ participate in the distributed skyline computation and each server $S_{i}$ can directly connect to any other server $S_{j}$. Each server $S_{i}$ stores locally a set of points $D_{i}$. The entire dataset $D$ is 
the union of all sets of points $D_{i}$ stored locally at any server $S_{i}\left(D=\bigcup D_{i}\right)$. A skyline query can be initiated by any server, henceforth also called query originator $S_{\text {org }}$. In such a distributed system, a skyline query is processed by sending the query to all servers $S_{i}$, which in turn process the query locally over their data $D_{i}$. Then, each server $S_{i}$ reports its local skyline set $S K Y_{i}$ to $S_{\text {org }}$ for subsequent merging (discarding of dominated local skyline points), in order to obtain the global skyline set $S K Y$. The correctness of the result is ensured by the property that the skyline points over a horizontally partitioned dataset are a subset of the union of the skyline points of all partitions $\left(S K Y \subseteq \bigcup S K Y_{i}\right)$.

Optimizing the performance of distributed skyline computation requires defining an appropriate execution plan. The execution plan defines the order in which the individual skyline queries are processed on different servers. By querying the servers consecutively, some servers may not have to be contacted at all, if all points of a server are dominated by a point stored locally at another server. Furthermore, the amount of transferred data can be drastically reduced. The local skyline points (or a fraction of them called filter points $[7,14] F_{i} \subseteq S K Y_{i}$ ) of a server $S_{i}$ can be used for discarding dominated points at another server $S_{j}$. In such a filter-based approach, the servers are accessed based on an execution order and the filter points of server $S_{i}$ are selected and transferred to the next servers $S_{j}$ to discard local skyline points produced by $S_{j}$. Filter points not only significantly reduce the amount of transferred data that need to be merged, but also the processing time at the subsequent servers by discarding points with few dominance tests only. However, the filter points may fail to prune any point of a server depending on the data distribution. When no gain can be obtained from querying the servers consecutively, the parallelism should be preserved, in order to minimize the latency and therefore also the response time ${ }^{1}$. To address this tradeoff, this paper introduces a novel framework, called SkyPlan, for processing distributed skyline queries that generates execution plans aiming at optimizing the performance of query processing.

Creation of efficient execution plans in distributed systems requires that the query originator $S_{\text {org }}$ has at least an abstract knowledge of the data stored on each server. Therefore, each server $S_{i}$ reports a set of minimum bounding rectangles ${ }^{2}$ (MBRs) to $S_{\text {org }}$ as a summarization of its data. Each MBR reported by a server $S_{i}$ defines a constrained skyline query on $S_{i}$, where the constraints are set by the boundaries of the MBR. Thus, $S_{\text {org }}$ generates an execution plan that defines the order of the constrained skyline queries on different servers.

$S_{\text {org }}$ can immediately discard dominated MBRs, since the local skyline points that belong to those MBRs are definitely dominated by points of other servers. The remaining MBRs are partitioned into incomparable groups, such that any two MBRs that belong to different groups are incomparable. Each incomparable group is processed in parallel, since no gain can be achieved by filtering. Unfortunately, the MBRs usually form only a few incomparable groups and thus, the number of MBRs in a group is high. This is because

\footnotetext{
${ }^{1}$ The response time is the time that passes between the query is posed until the results can be reported back to the user.

${ }^{2} \mathrm{An}$ MBR is a hyper-rectangle and any point of $S_{i}$ is enclosed by at least one MBR
}

the probability of partial dominance between any two MBRs is high and it increases with dimensionality. Therefore, the major challenge is establishing the execution order for the MBRs that belong to the same group. SkyPlan maps the dominance relationships between MBRs to a weighted directed graph, called skyline dependencies graph (SD-graph). The weights on the graph edges are defined by the pruning power, which is used to quantify the potential gain through filtering. Then, the SD-graph is transformed into an execution plan that maximizes the total pruning power, while preserving the parallelism, when no significant gain can be obtained from processing the queries on different servers consecutively. Furthermore, SkyPlan supports multi-objective executions plans, in case that additional objectives - other than maximizing the pruning power - need to be fulfilled simultaneously.

To summarize, SkyPlan consists of three phases: $S_{\text {org }}$ collects the MBRs of all servers $S_{i}$ and builds a weighted directed graph (Section 5); the graph is transformed into an execution plan that defines the order of query execution on servers (Section 6 and Section 7); and the plan is executed in a distributed manner that enables refinement of both the execution plan and the filter points during query processing (Section 8).

\section{SKYLINE DEPENDENCIES GRAPH}

In this section, we first provide the necessary definition of the graph that captures the dependencies between the skyline queries. Thereafter, we present the construction algorithm for the dependencies graph.

\subsection{Definition}

Given two constrained skyline queries defined by the MBRs $m_{i}$ and $m_{j}$, we say that there exists a dependency between them, if some points of $m_{i}$ may be dominated by points of $m_{j}$ or vice-versa. Thus, the dominance relationships between any pair of MBRs also define the dependency between the corresponding constrained skyline queries. SkyPlan maps the complex dependencies among a set of non-dominated MBRs to a graph, mentioned as skyline dependencies graph or $S D$-graph for short.

Definition 4. SD-graph G. Given a set of MBRs, the $S D$-graph is the weighted directed graph $G=(N, E, w)$, where:

- $N$ is the set of nodes and each node $n_{i} \in N$ corresponds to a non-dominated MBR $m_{i}$.

- $E$ is the set of edges. $E$ is a set of ordered pairs $e_{i j}=(i, j)$, where $m_{i}, m_{j}$ are MBRs, and $m_{i}$ partially dominates $m_{j}$.

- $w$ is a weight function defined as: $w_{i j}=\frac{\left|m_{j}\right|}{|D|} P P_{i j}$, where $\left|m_{j}\right|$ denotes the number of points enclosed in $m_{j},|D|$ the cardinality of the dataset $D$ and $P P_{i j}$ denotes the pruning power of $m_{i}$ on $m_{j}$.

Each non-dominated MBR $m_{i}$ is represented as a node (vertex) $n_{i}$ of the graph. Dominated MBRs are immediately discarded, as they enclose data points that are dominated and cannot belong to the skyline set. For each MBR $m_{j}$ that is partially dominated by $m_{i}$, a weighted directed edge $e_{i j}$ from node $n_{i}$ to $n_{j}$ is added. Consider the set of MBRs 


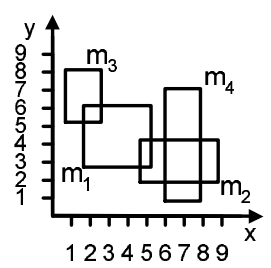

(a) Set of MBRs.

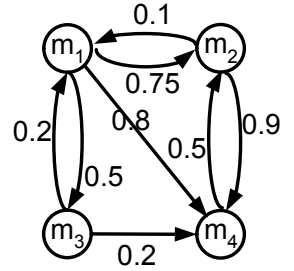

(b) SD-graph.
Figure 3: Construction of SD-graph.

depicted in Figure 3(a). In this example, $m_{1}$ partially dominates $m_{2}$, because the lower left corner of $m_{1}$ dominates the upper right corner of $m_{2}$. Thus, a directed edge from $m_{1}$ to $m_{2}$ is added to the graph (Figure 3(b)). Note that we use node $n_{i}$ and MBR $m_{i}$ interchangeably in our examples. The weight $w_{i j}$ of the edge $e_{i j}$ is defined as the pruning power $\left(P P_{i j}\right)$ of $m_{i}$ on $m_{j}$ normalized by the number of points $\left|m_{j}\right|$ enclosed in $m_{j}$ divided by the cardinality of the entire dataset $|D|$. The normalization is due to the fact that if $m_{i}$ has the same pruning power on two different MBRs, then the dependency between $m_{i}$ and the MBR that has more enclosed data points is more important for the overall performance of the distributed query.

\subsection{Construction of the SD-Graph}

The SD-graph is constructed by comparing each pair of MBRs. If an MBR is dominated, its node is removed from the graph. If an MBR is partially dominated, then an edge is added between the corresponding nodes.

Algorithm 1 presents our algorithm for the construction of the SD-graph. Given a set of MBRs, our algorithm constructs the SD-graph incrementally, by adding to the graph one MBR in each iteration. Initially, the sets of nodes and edges of the SD-graph are empty. Then, the algorithm takes as input the current form of the SD-graph $G$ and a new MBR $m_{n}$, which should be accommodated in the existing graph $G$. Thus, a new node is added to the graph $G$ that represents the MBR $m_{n}$ (line 3). Then, each node of $G$ (corresponding to MBR $m_{i}$ ) is compared against $m_{n}$ for dominance, in order to discard dominated MBRs (lines 5,9). If $m_{n}$ is dominated by or dominates $m_{i}$, then the dominated MBR is discarded. Once an MBR is dominated, all edges connected to the discarded MBR are also eliminated.

Otherwise, $m_{n}$ and $m_{i}$ are tested for partial dominance, in order to determine potential dependencies and add the corresponding edges. If $m_{i}$ partially dominates $m_{n}$ (line 14), an edge from $m_{i}$ to $m_{n}$ is created (line 15). In the same spirit, if $m_{n}$ partially dominates $m_{i}$ (line 17), a directed edge from $m_{n}$ to $m_{i}$ is added (line 18). Note that when an edge is added to the graph, the weight of the edge is also computed. For sake of simplicity, this is not explicitly shown in the algorithm. At the end of this process, the constructed SD-graph accurately captures all dependencies between any pair of MBRs.

\section{COST-AWARE EXECUTION PLANS}

In this section, we first provide the definitions of execution plan and its quality in terms of pruning power. Then, we present an algorithm which maps the SD-graph into the execution plan that has the maximum pruning power.

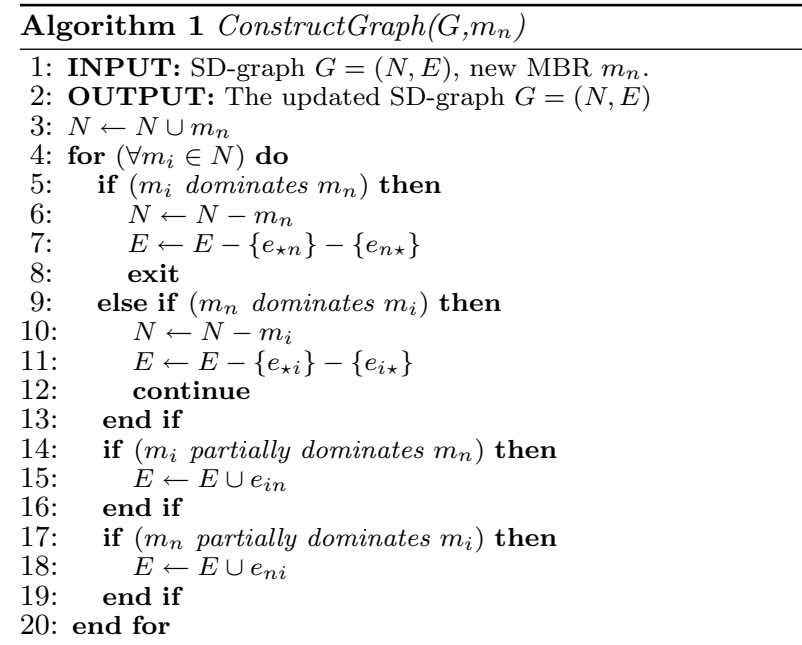

\subsection{Definitions}

During distributed skyline query computation an execution plan defines the order of execution of the constrained skyline queries on the individual servers.

DEFINITION 5. Execution plan. Given a distributed skyline query, an execution plan $P(N, E)$ is a set of directed rooted weighted trees (forest), where the nodes $N$ represent the individual constrained skyline queries processed on different servers and the weights of edges $E$ represent the pruning power associated with pairs of skyline queries.

A first observation is that the execution plan is either a tree or a set of trees. The edges have a natural orientation away from the roots. Each tree is processed in parallel and the children of a node are processed after the parent node. Thus, the fan-out of a node defines the degree of parallelism, while the child-parent relation reduces the communication cost of the skyline computation via filtering. The weight that is assigned to an edge $e_{i j} \in E$ indicates the strength of the dependency between the corresponding queries and quantifies the gain of processing the skyline query on $n_{j} \in N$ after $n_{i} \in N$. As discussed in Section 5 , the pruning power of a edge is an appropriate measure to quantify the potential gain of consecutive queries. Thus, the quality of an execution plan is defined as follows.

DEFINITION 6. Quality of execution plan. The quality $Q(P)$ of an execution plan $P(N, E)$ is defined as the sum of weights on the edges of $P$ :

$$
Q(P)=\sum_{e_{i j} \in E} w_{i j}
$$

Thus, an execution plan $P_{i}$ is better than another plan $P_{j}$ in terms of quality if: $Q\left(P_{i}\right)>Q\left(P_{j}\right)$.

A straightforward execution plan, also called linear in [7], is to execute queries in a sequential manner, by defining an appropriate ordering of queries ${ }^{3}$. Even though the linear approach reduces the transferred data through filter points, it does not exploit parallelism at all, and fails to process in parallel even queries that are independent. Furthermore, the dependencies among the constrained skyline queries form a

${ }^{3}$ In [7] the linear execution plan uses the Euclidean distance from the origin for sorting. 


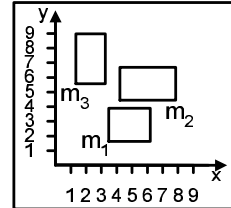

(a) MBRs.

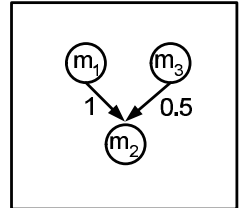

(b) SD-graph.

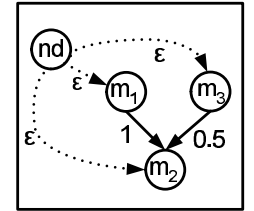

(c) Dummy node.

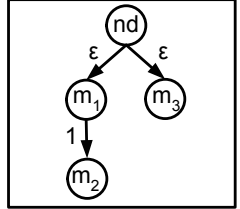

(d) Spanning tree.

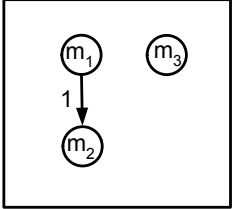

(e) Plan.
Figure 4: Example of dummy node.

complex graph (SD-graph) that contains cycles and multiple incoming edges to some nodes. Therefore, the mapping of the SD-graph to any execution plan leads to substantial loss in terms of overall pruning power, as several edges cannot be taken into account. Thus, even for a linear execution plan, the efficiency in terms of pruning power is not guaranteed. In the following, we define an execution plan that is generated from the SD-graph and induces sequential processing of queries with the most significant (in terms of pruning power) dependencies, and parallelizes query execution in other cases.

Notice that the SD-graph can in general be disconnected and therefore may consist of more than one connected components (mentioned as subgraphs), such that no edge connects any two nodes that belong to different subgraphs. In this case, the connected subgraphs of the SD-graph correspond to the incomparable groups of MBRs (as discussed in Section 4). Therefore, an individual plan is created (for each subgraph), which is executed in parallel and their results are reported independently. Henceforth, we assume that the $\mathrm{SD}$-graph is connected and present our algorithm for generating query execution plans. Obviously, if the SD-graph is disconnected, the algorithm is applied on each connected subgraph of the SD-graph.

\subsection{Maximizing the Pruning Power}

Based on Definition 6, an execution plan is better than another execution plan, if its quality in terms of pruning power is higher. Consequently, the ideal execution plan is the execution plan with the maximum pruning power.

DEFINITION 7. Maximum pruning execution plan. Let $\Pi$ denote the set of all possible execution plans. The maximum pruning execution plan $\hat{P}$ is the one that maximizes the quality of any possible execution plan $P \in \Pi$ :

$$
\hat{P}=\arg \max _{P \in \Pi} Q(P)
$$

The problem of generating the maximum pruning execution plan from the SD-graph is equivalent to the maximum spanning tree problem, which is formulated as follows.

Problem 1. The Directed Maximum Spanning Tree Problem. Consider a directed graph, $G(N, E)$, where $N$ and $E$ are the sets of nodes and edges respectively. Associated with each edge $e_{i j} \in E$ is a weight $w_{i j}$. Given a node $n_{r}$, the problem is to find a rooted directed spanning tree $P\left(N, E^{\prime}\right)$ with root $n_{r}$, where $E^{\prime}$ is a subset of $E$, such that the sum of $w_{i j}$ for all $e_{i j} \in E^{\prime}$ is maximized. The rooted directed spanning tree is defined as an acyclic graph that connects all nodes using $|N|-1$ edges, which means that each node (excluding the root) has one and only one incoming edge.

A prerequisite for the existence of a directed rooted spanning tree of the SD-graph is that all nodes must be reachable from the root node, which means that there must exist a directed path from the root to any other node $n_{i}$. This may not hold for any node in the SD-graph, even though the SDgraph is connected. Consider the small example with three MBRs depicted in Figure 4(a). The SD-graph has two edges (Figure $4(\mathrm{~b})$ ), i.e., $m_{1} \rightarrow m_{2}$ and $m_{3} \rightarrow m_{2}$. For this graph, there does not exist a directed tree that connects all nodes, no matter which node is selected as a root. Therefore, it is not possible to create an execution plan that consists of one directed tree from this graph and the only feasible solution is a set of directed rooted trees.

In order to ensure that there always exists a solution for the directed maximum spanning tree problem, we use the following technique. A dummy node $n d$ is added to the graph, which is directly connected with all other nodes. All (dummy) edges that start from the dummy node have the same weight, equal to a very small value $\varepsilon$ (Figure $4(\mathrm{c})$ ). By using the dummy node as the root for the maximum spanning tree problem, there always exists a directed rooted tree that is a solution of the problem, since all nodes become reachable from the root. Furthermore, a dummy edge is selected only in the case where there exists no directed spanning tree with fewer dummy edges. After the generation of the spanning tree (Figure 4(d)), the dummy node is discarded from the execution plan. After the removal of the dummy node, the execution plan consists of two trees $m_{3}$ and $m_{1} \rightarrow m_{2}$ (Figure 4(e)). Notice that the potential pruning of $m_{2}$ by data points in $m_{3}$ cannot be established, since the execution plan cannot maintain all dependencies, due to the cycles and multiple incoming edges that exist in the SD-graph. The definition of the maximum spanning tree problem guarantees that the produced execution plan has the highest pruning power as there exists no other treebased plan with higher value of pruning power. The usage of the dummy node ensures that there always exists a spanning tree and also solves the problem of finding an appropriate node for the root of the spanning tree.

Edmonds algorithm. To derive the maximum directed spanning tree of a graph $G$, we employ the algorithm of Edmonds $[6,8]$. In the sequel, we briefly sketch Algorithm 2. The algorithm takes as input a node $v_{\text {root }}$ that is the root of the spanning tree. In our case, the root of the spanning tree is always the dummy node. First, for each node, except for the root, the incoming edge with the maximum weight is selected. Therefore, $|N|-1$ edges are selected in set $S$, as many as the edges of the spanning tree (lines 3-6). If there does not exist a cycle, the algorithm terminates by returning the spanning tree. Otherwise, the main idea of the algorithm is to add those edges that lead to minimum loss in terms of weights, compared to the weights of the edges that form the cycle. Thus, for each cycle, an edge $e_{k j}$ of the cycle pointing to a node $v_{j}$ is removed and replaced by an unselected edge $\left(e_{i j} \in E\right)$ pointing to $v_{j}$ (line 15). To this end, all edges pointing to a node that belongs to the cycle are examined and inserted in set $M$ (lines 9-13). The edge 


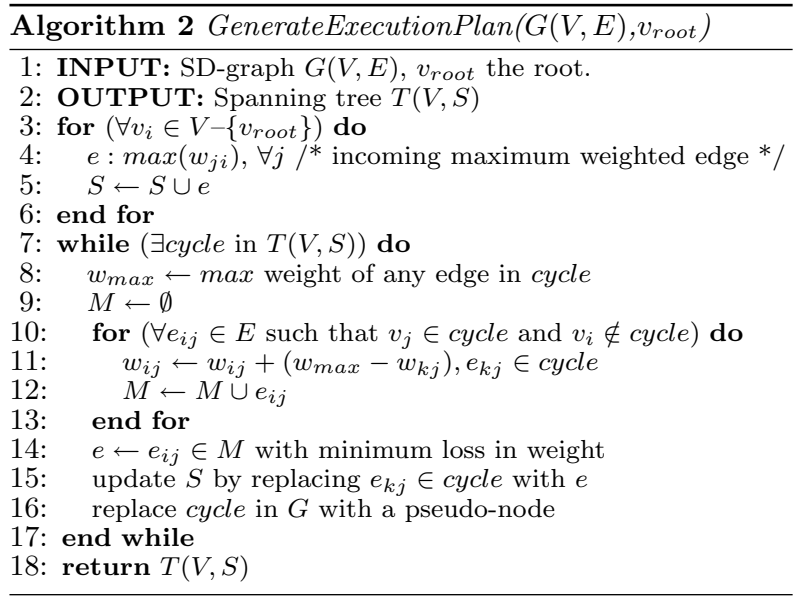

in $M$ that minimizes the loss in weights is selected (line 14), which is the edge with maximum $w_{i j}$ (as computed in line 11). Furthermore, in each iteration the cycle is replaced by a pseudo-node (line 16) and the weights pointing to the cycle are modified (line 11). This ensures that parts of the graph $G$ that have been mapped to a subtree by removing a cycle, are not examined further. The algorithm terminates when all cycles are eliminated. This algorithm is a polynomialtime algorithm for finding a maximum directed spanning tree. A more efficient implementation of the algorithm with complexity $O(|E|+|N| \log |N|)$ is presented in [11].

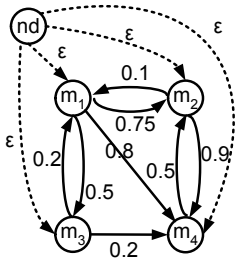

(a) Dummy node.

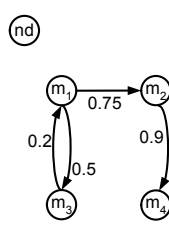

(b) Step 1.

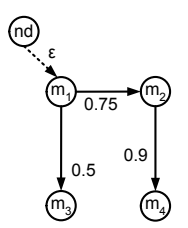

(c) Step 2.

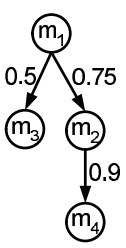

(d) Plan.
Figure 5: Construction of the execution plan.

Example 1. Figure 5 depicts the process of generating the execution plan that corresponds to the SD-graph of Figure 3(b). First, the dummy node is added to the SD-graph (Figure 5(a)). Then, in the first iteration of Algorithm 2 the edges depicted in Figure 5(b) are selected. Since there exists a cycle, the edge pointing from $m_{3}$ to $m_{1}$ is replaced with the edge from nd to $m_{1}$ (Figure 5(c)). Afterwards, Algorithm 2 terminates, since there exists no other cycle. Finally, the dummy node is removed leading to the execution plan depicted in Figure 5(d). Based on the definition of the maximum spanning tree for the given SD-graph, there exists no other execution plan with higher sum of the edges' weights than the depicted execution plan. The node $m_{1}$, which has a high dependency to $m_{2}$ and $m_{3}$, is the root node of the execution plan. On the other hand, the MBRs $m_{2}$ and $m_{3}$ are processed in parallel without any loss of pruning power since they are incomparable. Nevertheless, in order to break the cycles that exist in the $S D$-graph, points of $m_{1}$ cannot be pruned by points of $m_{2}$ nor $m_{3}$ based on the execution plan, since $m_{1}$ has a higher pruning power on $m_{2}$ and $m_{3}$.

\section{MULTI-OBJECTIVE EXECUTION PLANS}

Depending on the characteristics or the current load of a distributed system, the generation of execution plans that satisfy additional objectives, apart from maximizing the total pruning power, is desirable. Such an objective is to restrict the number of hops that the query has to be forwarded, so that the latency of the system is bounded and the overall execution time is improved. Another objective is to share the processing load fairly to the servers, by producing balanced execution plans, so that bottlenecks and single points of failure are avoided. In this section, we extend SkyPlan to a generic framework that supports a variety of execution plans in order to adapt to the requirements of different distributed systems.

\section{1 k-Hop Execution Plan}

A major concern in many distributed systems is bounding the number of hops that are required for query processing. This is particularly important for many applications that require low latency during query processing. The number of required hops is determined by the height of the execution plan. To bound the number of hops, we define the $k$-hop execution plan and show that the SkyPlan framework can be adapted to support such plans efficiently.

Definition 8. k-hop execution plan. Given a constraint of $k$ hops, the $k$-hop execution plan $\hat{P}_{k}$ is the execution plan with height at most $k$ (height $\left(\hat{P}_{k}\right) \leq k$ ) that has the maximum quality $Q\left(\hat{P}_{k}\right)$ among all execution plans with height at most $k$.

The problem of producing a $k$-hop execution plan is equivalent to the hop-constrained maximum spanning tree problem that produces the spanning tree with height at most $k$ that has the maximum sum of edges' weights. This problem is NP-Hard [12], therefore we employ a heuristic algorithm to produce the $k$-hop execution plan efficiently. Specifically, we adapt a solution proposed by Abdalla et al. [1] for the diameter-constrained minimum spanning tree problem, since the hop-constrained maximum spanning tree problem is a simplification of the bounded-diameter minimum spanning tree problem [15]. Notice that the dummy node added to the SD-graph ensures that there always exists at least one spanning tree with maximum height $k(k \geq 1)$.

The algorithm that builds the $k$-hop execution plan works as follows. First, the maximum pruning execution plan is created by applying Algorithm 2 on the SD-graph. If the length of the longest path is at most $k$, then the generated execution plan is also the k-hop execution plan and our algorithm terminates. Otherwise, the generated execution plan is modified by reducing the height of the tree iteratively until its maximum path length is at most $k$. During each iteration, the tree is traversed and the longest path of the tree is detected. Then, the edge with minimum pruning power (excluding dummy edges) in the path is replaced with another edge of SD-graph that reduces the path in at least one hop. Notice that in our case any edge of the plan can be replaced by a dummy edge that reduces the length of the path, because the dummy node is connected to all nodes. If more than one edge that reduce the length of the path exist, then the edge with the highest pruning power is selected. In the next iteration, the current longest path is selected and its length is reduced, until the length of the longest path is at most $k$. 


\subsection{Capacity Constrained Execution Plan}

Another desirable feature of distributed query processing is to balance the load fairly to all participating servers. Load balancing is important in order to avoid bottlenecks in the system when the query workload increases. To this end, we define the capacity constrained execution plan that aims to balance the load, and we describe the process of generating such balanced plans by SkyPlan.

DeFINITION 9. Capacity constrained execution plan. Given a capacity b, an execution plan satisfies the capacity constraint, if every subtree $s$ of the root node has at most $b$ nodes (size $(s) \leq b)$. The capacity constrained execution plan $\hat{P}_{b}$ is the execution plan that has the maximum quality $Q\left(\hat{P}_{b}\right)$ among all execution plans that satisfy the capacity constraint.

The problem of producing a capacity constrained execution plan is equivalent to the problem of computing a capacitated maximum spanning tree (CMST) [2]. In the general case of CMST, both edges and nodes have weights and the capacity of a subtree is the sum of the weights of all nodes in the subtree. The CMST is the problem of finding the spanning tree with maximum quality that satisfies the capacity constraint for all subtrees. One interesting instance of the CMST problem is when all weights of the nodes are set to one (except of the weight of the root node that is set to zero). This problem is known as equal weight CMST and it has also been shown to be NP-hard [2]. The equal weight CMST problem can be employed for finding the capacity constrained execution plan. We employ an approximate algorithm [9] to obtain the execution plan efficiently. The existence of the dummy node again guarantees that the SD-graph can always produce such a plan.

The algorithm starts with generating the maximum spanning tree using Algorithm 2 and then checks if the produced execution plan satisfies the capacity constraint. If the constraint is satisfied, no modification is needed and the algorithm terminates. If not, the algorithm separates the nodes $n_{i}$ of the generated execution plan in two sets $A$ and $B$. The set $A$ contains all nodes that are in subtrees that satisfy the capacity constraint, while the set $B$ contains the remaining nodes. The algorithm then selects the edge $e_{i j}$ with smallest weight such that $n_{j} \in B$. This edge is replaced with another edge $e_{l j}$ of the SD-graph such that (a) $n_{l} \in A$, and (b) the replacement causes the reduction of the overall capacity of the plan. The algorithm terminates when all subtrees have at most $b$ nodes.

\section{DISTRIBUTED QUERY PROCESSING}

Any server in the system can pose a skyline query and the querying server is referred to as query originator $S_{\text {org }}$. The query execution starts with $S_{\text {org }}$ requesting in parallel the MBRs of all servers $S_{i}$. Each $S_{i}$ reports a set of MBRs to $S_{\text {org }}$, and while the MBRs from the servers $S_{i}$ are received, $S_{\text {org }}$ builds the SD-graph $G(N, E)$ using the incremental construction algorithm (Algorithm 1). The incremental property of the algorithm ensures that $S_{\text {org }}$ does not need to wait until it receives all MBRs, but starts as soon as it receives MBRs from any server $S_{i}$. After receiving all MBRs and building the SD-graph, an appropriate execution plan is established as described in Sections 6 and 7.

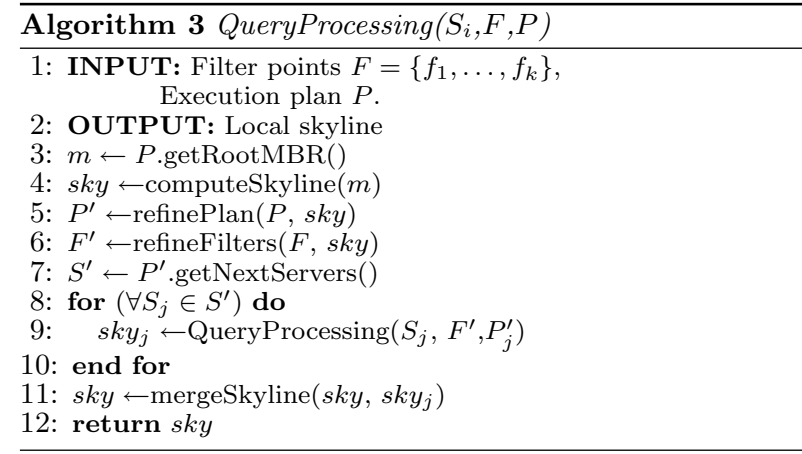

The distributed skyline query is processed based on the execution plan. The query originator $S_{\text {org }}$ sends a skyline query to the root of every directed tree in the execution plan. The query sent to a server $S_{i}$ consists of the execution plan and a set of $k$ filter points $F=\left\{f_{1}, \ldots, f_{k}\right\}$. Initially, the set of filter points $F$ is empty.

\subsection{Query Execution and Plan Refinement}

Query execution on a server $S_{i}$ is initiated when the execution of the plan reaches an MBR $m_{j}$ of $S_{i}$. This initiates a constrained skyline query on $m_{j}$. Server $S_{i}$ takes as input a set of filter points $F$ and the execution $\operatorname{plan}^{4} . S_{i}$ produces as output the local skyline points, computes a new set of filter points and refines the execution plan, as will be explained shortly. Then, $S_{i}$ forwards the query to the servers that are next based on the refined execution plan.

Algorithm 3 provides the pseudocode for query processing on server $S_{i}$ that received a skyline request. Initially, $S_{i}$ processes locally the constrained skyline query (line 4 ) defined by the MBR $m$ that is the root of execution plan $P$ (line 3 ). The locally computed skyline set with constraint $m$ is denoted as sky. Then, $S_{i}$ refines the execution plan (line 5), by removing MBRs that are dominated by one of the local skyline points sky. Notice that $S_{i}$ also removes the current MBR from the execution plan. In addition, $S_{i}$ is able to refine the filter points $F^{\prime}$ (line 6), by taking into account the local skyline points and the filter points in the set $F$. Finally, $S_{i}$ determines the next set of servers $S^{\prime}$ that should process the query based on the refined plan $P^{\prime}$ (line 7), and sends a skyline query to each of them (line 9), passing as arguments the refined filter points $F^{\prime}$ and the refined plan $P^{\prime}$. Each server $S_{j}$ receives a different execution plan $P_{j}^{\prime}$ that corresponds to the part of the execution plan that has as a root the MBR of the server $S_{j}$. Eventually, $S_{i}$ gathers the local result sets of the servers $S_{j}$ (that had received the query through $S_{i}$ ) and merges them by discarding dominated points (line 11). Local processing on server $S_{i}$ terminates by returning the local merged skyline points to the previous server based on the execution plan.

The refinement of the execution plan $P$ at server $S_{i}$ produces a new execution plan $P^{\prime}$ that does not contain the nodes (representing MBRs) that are dominated by the local skyline points of $S_{i}$. In order to maintain the connectivity of the execution plan, when a node is removed, its children nodes substitute it in the plan. For an illustrative example see Figure 6(a), which depicts an execution plan $P$ (left)

${ }^{4}$ Henceforth, we will refer to the subtree of the execution plan that $S_{i}$ receives as execution plan at $S_{i}$. 

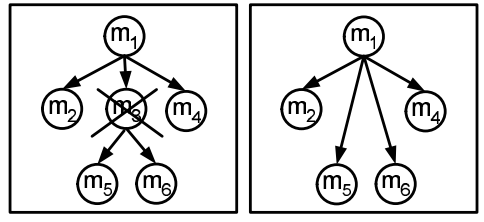

(a) Plan refinement example.

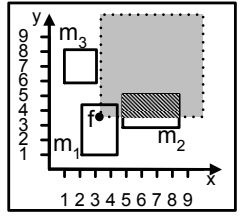

(b) Filter points.

Figure 6: Plan refinement and filter points.

and a refined plan $P^{\prime}$ (middle). Assume that server $S_{i}$ determines that $m_{3}$ is dominated by local results and should therefore be discarded. In the refined execution plan $P^{\prime}$, the children MBRs of $m_{3}$, namely $m_{5}$ and $m_{6}$, substitute $m_{3}$.

Essentially, as the execution plan is forwarded to servers during query processing, a distributed query execution mechanism is established, which is not coordinated rigidly by one server. Instead, it is a loose approach in which each server is assigned not only the task of local query processing, but also the refinement of the execution plan and the continuation of query processing.

\subsection{Filter Point Selection and Refinement}

SkyPlan discovers an appropriate execution order to execute the constrained skyline queries on servers $S_{i}$ by using the pruning power. The filter points facilitate pruning of local data points, hence the selection of the $k$ filter points $F=\left\{f_{1}, \ldots, f_{k}\right\}$ influences the overall performance. The filter points are selected from the points that belong to local result set and the previous filter points. We refer to these points as candidate filter points.

In [7], the volume of the dominance area is used to estimate the pruning power of a potential filter point. The main shortcoming of this approach is that the volume of the dominance area does not necessarily relate to the area within an MBR that is dominated by a filter point. For example, in Figure 6(b), even though filter point $f$ has a high volume of dominance area, the actual area dominated in $m_{2}$ is much smaller. Furthermore, for different MBRs, different filter points may be appropriate. Again in Figure 6(b), point $f$ is not appropriate for $m_{3}$ since it cannot dominate any point enclosed in $m_{3}$, although it prunes points in $m_{2}$.

In order to alleviate such deficiencies, we propose two different methods for filter point selection that rely on the enclosed dominance area of filter point $f$. In Figure 6(b), the enclosed dominance area of filter point $f$ on $m_{2}$ is depicted as the dashed area. Obviously, the enclosed dominance area quantifies the pruning power of a candidate filter point in a more accurate way by taking into account the MBRs of the execution plan.

Our filter strategies discover the filter points that maximize the pruning power based on the queries that will be processed in the next step. Therefore, given an execution plan, the MBRs corresponding to the children of the current node are taken into consideration. The first strategy selects as refined filter points the set of $k$ candidate points that maximize the total enclosed dominance area for all children MBRs. Therefore, for each candidate filter point $f_{i}$, the volume of enclosed dominance area is computed for each child MBR $m_{j}$ and the total volume is defined as the sum of the volumes. Thus, the first strategy selects the same set of filter points for all children MBRs of the current node in the execution plan. The second strategy refines the filter points

even further, by defining a different set $F_{i}$ of filter points for each child MBR. In this case, for each MBR $m_{i}$ the $k$ filter points with the highest volume of the enclosed dominance area in $m_{i}$ are selected.

\section{EXPERIMENTAL EVALUATION}

In this section, we provide an experimental evaluation on the performance of SkyPlan. SkyPlan was implemented in Java, while the network aspects were simulated using DesmoJ $^{5}$, an event-based simulator framework. All experiments were conducted on a PC equipped with a $3 \mathrm{GHz}$ Dual Core AMD processor and 2GB RAM. The parameters and values used in our experiments are outlined in Table 1 (the values in bold are the default values).

\begin{tabular}{|l|l|}
\hline Parameter & Values \\
\hline Dimensions & $3, \mathbf{4}, 5,6$ \\
\hline Number of servers & $\mathbf{1 K}, 2 \mathrm{~K}, 3 \mathrm{~K}, 4 \mathrm{~K}$ \\
\hline Cardinality of each server & $\mathbf{1 K}, 2 \mathrm{~K}, 3 \mathrm{~K}, 4 \mathrm{~K}$ \\
\hline Data distributions & $\mathrm{RL}, \mathbf{C L}, \mathrm{UN}, \mathrm{CO}, \mathrm{AC}$ \\
\hline Network speed (Mbit/s) & $0.05, \mathbf{0 . 2}, 0.8,3.2,12.8$ \\
\hline
\end{tabular}

Table 1: Experimental parameters and values.

We employed both synthetic and real datasets. For the synthetic datasets we examined different distributions, namely uniform (UN), clustered (CL), correlated (CO), and anticorrelated (AC). For the clustered dataset (CL), each server picks cluster centroids randomly and the points follow a Gaussian distribution on each axis with variance 0.025 , and a mean equal to the corresponding coordinate of the centroid. The correlated (CO) and anticorrelated (AC) datasets were generated as described in [4]. For our experiments on synthetic data, we report the average results over 20 different instances of the data set. We generate the different instances by keeping the parameters fixed and changing the seeds of the random number generator. We adopt this approach in order to factor out the effects of randomization. The real dataset (RL) contains information about real estate all over the United States (crawled from http://www.zillow.com/). It is a 5 -dimensional dataset containing more than $2 \mathrm{M}$ entries with the following attributes: number of bathrooms, number of bedrooms, living area, price and lot area. In all cases, the dataset is horizontally partitioned evenly among the servers. In our experiments, we set the maximum number of filter points $k$ equal to 5 , but $k$ does not exceed the $10 \%$ of the local skyline points.

Our main metrics are: (i) the response time, which is the total time until the final result is produced at $S_{\text {org }}$ (including the time for generating the execution plan), and (ii) the amount of transferred data. In addition, we measure the Data Reduction Rate (DRR), which has been used in both [7] and [14]. The DRR at a server $S_{i}$ is measured using the following equation:

$$
D R R_{i}=\frac{\left|S K Y_{i}\right|-\left|S K Y_{i}^{R}\right|-\left|F_{i}\right|}{\left|S K Y_{i}\right|}
$$

where $\left|S K Y_{i}\right|$ is the cardinality of the skyline of $S_{i}$ without filtering, $\left|S K Y_{i}{ }^{R}\right|$ is the cardinality of the skyline of $S_{i}$ after filtering, and $\left|F_{i}\right|$ is the cardinality of the filter point set received by $S_{i}$. The DRR quantifies the gain in transferred data that is obtained when filter points are used.

In Section 9.1, we compare SkyPlan against the state-ofthe-art algorithm (PaDSkyline [7]), which has two variants:

${ }^{5} \mathrm{http} / / /$ desmoj.sourceforge.net/home.html 


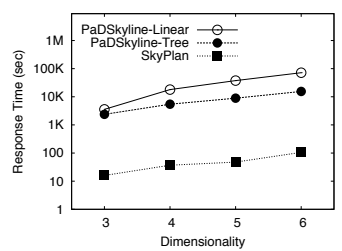

(a) Time vs. dimensionality.

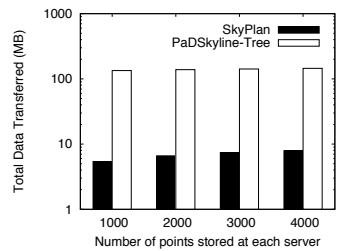

(b) Data vs. cardinality.

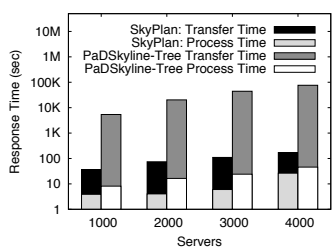

(c) Time vs. servers.

Figure 7: Comparative performance of SkyPlan against PaDSkyline for uniform (UN) data distribution.

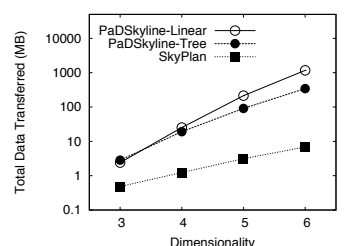

(a) Data vs. dimensionality.

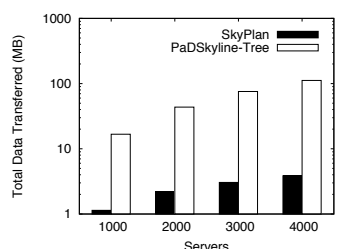

(b) Data vs. servers.

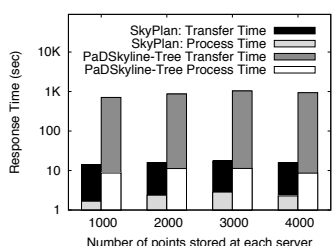

(c) Time vs. cardinality.

Figure 8: Comparative performance of SkyPlan against PaDSkyline for clustered (CL) data distribution.

Linear and Tree. PaDSkyline-Tree constructs an execution plan in which the order of accessing servers depends on the order that the MBRs are received by $S_{\text {org }}$. We implemented PaDSkyline in the same framework as SkyPlan with recursive data transfer according to the execution plan. In addition, we evaluate the different filter point strategies in Section 9.2, and compare the performance of the multi-objective execution plans to the maximum pruning execution plan in Section 9.3. We emphasize that the maximum pruning execution plan is used as the default execution plan of SkyPlan in our experimental evaluation.

\subsection{Comparative Performance Study}

In Figures 7, 8 and 9 we present a thorough comparative study of SkyPlan against PaDSkyline for miscellaneous setups and different data distributions. We assess the performance of both approaches using the same filter strategy in order to compare the produced execution plans directly, without interference from other factors. Notice that the yaxis is in logarithmic scale in all these charts.

First, we assess the performance of SkyPlan for uniform data distribution in Figure 7. In order to test the scalability of SkyPlan, we vary the dimensionality (Figure 7(a)), the number of points stored at each server (Figure 7(b)), and the number of servers (Figure 7(c)). In Figure 7(a), we compare the response time of SkyPlan against PaDSkyline. SkyPlan is 2-3 orders of magnitude better than PaDSkyline-Tree, and this gain is sustained with increased dimensionality. This result verifies that the execution plan deployed by SkyPlan improves the performance of query processing. The height of the generated plans are 6-11 in all setups thus verifying that it does not degenerate to the linear execution plan (figures are omitted due to lack of space). As expected, the response time required by PaDSkyline-Linear is even higher, which deems it impractical for distributed settings. Hence, it is omitted in the following charts.

Then, in Figure 7(b), we depict the number of total transferred data by SkyPlan compared to PaDSkyline-Tree for varying the number of points per server. In all cases, Sky-
Plan transfers more than 1 order of magnitude fewer data. This indicates that SkyPlan defines the execution order of the queries in such a way that the amount of transferred data is reduced. In Figure 7(c), we study the response time for varying number of servers and we provide a cost breakdown analysis of the response time, in order to clearly illustrate the effect of networking and processing cost. The chart shows that the total gain in response time is both due to the reduction of transferred data (networking cost) as well as the reduction of processing time (plan generation, skyline computation and result merging). Moreover, notice that SkyPlan's processing cost for the generation of the execution plan is amortized over the cost of query execution. We also investigate the performance of SkyPlan against both variants of PaDSkyline for clustered data distribution in Figure 8. Again, we observe that SkyPlan shows consistently better performance than PaDSkyline for all evaluated metrics and for different setups.

Furthermore, in Figure 9, we evaluate the response time and total transferred data of SkyPlan and PaDSkyline for different data distributions. This experiment includes additionally correlated, anti-correlated and real data. We notice that SkyPlan achieves better processing and transfer time (Figure 9(a)) regardless of the data distribution. Even in the case of the demanding anti-correlated dataset, SkyPlan is almost 2 orders of magnitude better than PaDSkyline. In Figure 9(b), we evaluate the total amount of transferred data. Again SkyPlan is much more efficient (usually more than 1 order of magnitude) in transferred data in all setups. In Figure 9(c), we vary the network speed, in order to verify that the gain in transfer time of SkyPlan is sustained. Obviously both approaches present almost constant processing time for varying network speed. Also, as expected, the transfer time is reduced with higher network speed for both approaches. The important finding is that the improvement of SkyPlan in transfer time is sustained both for lower and higher transfer rates. This is a strong argument in favor of SkyPlan, as its performance gains are attained irrespective of the network speed. 


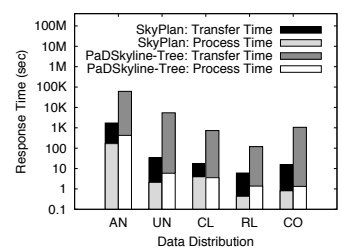

(a) Time vs. distribution.

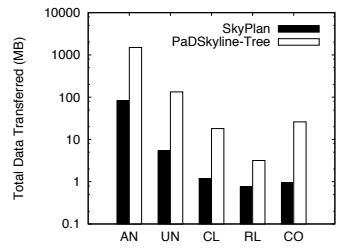

(b) Data vs. distribution.

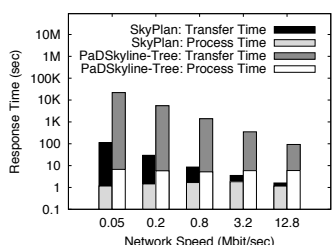

(c) Time vs. network speed.

Figure 9: Comparative performance for different data distributions and network speed (UN distribution).

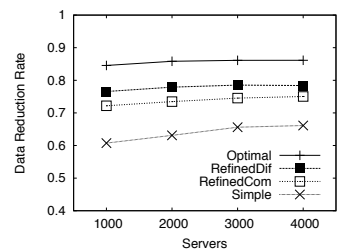

(a) DRR for clustered data

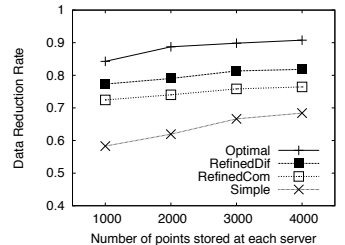

(b) DRR for clustered data.

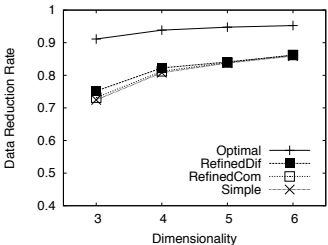

(c) DRR for uniform data.

Figure 10: Data reduction rate (DRR) for different filter point strategies.

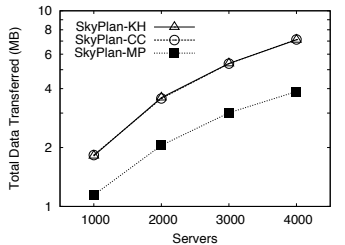

(a) Data.

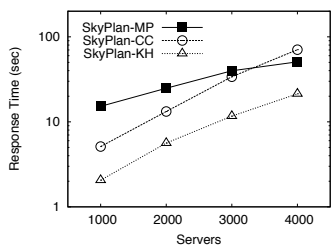

(c) Time.

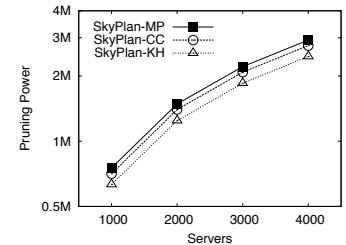

(b) Pruning power.

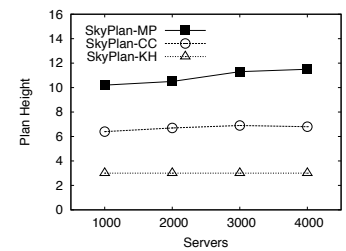

(d) Height.
Figure 11: Multi-objective execution plans for varying number of servers.

\subsection{Effect of Filter Points}

In the next series of experiments, we focus on the filter strategy and compare the different strategies using Data Reduction Rate (DRR). We also define a theoretical optimal filter strategy that is an upper bound of DRR that can be achieved. The optimal strategy assumes that the complete skyline $S K Y_{i}$ and the complete set of received filter points $F_{i}$ are sent to the next servers $S_{j}$. In this case the filter points received by $S_{j}$ are $F_{j}=S K Y_{i} \bigcup F_{i}$. By transferring all these points, we capture the maximum pruning power possible. In order to define an optimal value for DRR, instead of using the $\left|F_{j}\right|$ during the DRR computation we use $\min \left(k,\left|F_{j}\right|\right)$. Thus, the optimal approach transfers all available points, while paying only for $k$ points as a cost. This optimal is not realistic nor feasible, but it still defines an upper bound for the DRR, thus establishing a ceiling for the performance of any algorithm.

Figure 10 presents the DRR for different filter strategies and various settings. In Figure 10(a) and Figure 10(b) we study the performance for clustered data distribution. Our strategy that sends the same filter points to all servers is denoted as RefinedCom, while RefinedDif denotes the case that different filter points are used for each server. We compare to the maxSum strategy proposed in [7], here denoted as Simple. Our strategies perform better than the competitor approach by $15 \%-33 \%$. It is noteworthy that the RefinedDif strategy manages to significantly reduce the gap to optimal and to achieve gains in transferred data that are close to the theoretical optimal. Moreover, our strategies scale with the number of servers (Figures 10(a)) and the cardinality on each server (Figure 10(b)). In terms of comparison of our strategies, we observe that RefinedDif outperforms RefinedCom, because RefinedDif refines the filter points for each MBR individually, thus achieving better pruning power. For uniform data distribution, presented in Figure 10(c), all approaches are comparable which is expected as data objects are uniformly distributed in the data space. However, all filter strategies achieve high DRR values.

\subsection{Multi-objective Execution Plans}

In this section, we evaluate the performance of multiobjective execution plans, namely the $k$-hop execution plan $($ SkyPlan-KH) and the capacity constrained execution plan (SkyPlan-CC), compared to the maximum pruning execution plan (SkyPlan-MP). The maximum number of hops for the SkyPlan-KH is set to 3 , while the maximum capacity for SkyPlan-CC is set to 20. In Figure 11, we compare the performance of the execution plans varying the number of servers for clustered data distribution. Figure 11(a) depicts the total transferred data for the different execution plans. SkyPlan-MP transfers fewer data than SkyPlan-KH and Skyplan-CC because its execution plan has the highest pruning power (Figure 11(b)). The high pruning power of the maximum pruning execution plan leads to filter points that prune more local skyline points. Consequently, the total transferred data is reduced. Figure 11(b) shows the total pruning power, i.e., the sum of edges' weights of the different execution plans. Clearly, there is a small loss in pruning 


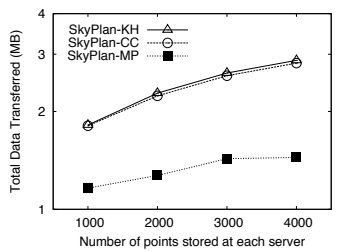

(a) Data.

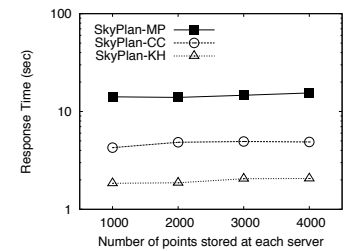

(b) Time.
Figure 12: Multi-objective execution plans for varying cardinality.

power for the multi-objective query plans caused by the imposed constraints. On the other hand, SkyPlan-KH presents the best performance in terms of response time, as depicted in Figure 11(c). The main reason is that the maximum number of hops required to process a skyline query is bounded by the fixed height of the execution plan. This leads to lower latency, thus reducing the response time. SkyPlan-CC generates balanced execution plans, which indirectly leads to plans with smaller height than SkyPlan-MP, because the capacity constrained execution plan avoids the existence of one long path. Therefore, SkyPlan-CC outperforms SkyPlan$\mathrm{MP}$ in terms of response time for small number of servers, but for higher number of servers (> 3000) SkyPlan-MP performs better. The height of the different execution plans is depicted in Figure 11(d).

Figure 12 presents a comparative performance of different execution plans varying the number of points stored at each server for clustered data distribution. Figure 12(a) depicts the total amount of transferred data for each execution plan. SkyPlan-MP transfers around $50 \%$ fewer data than the other plans. This verifies that an execution plan with high pruning power reduces the amount of transferred data. In Figure 12(b), we evaluate the response time of the different execution plans. SkyPlan-KH achieves the smallest response time followed by Skyplan-CC. Nevertherless, the performance of all approaches is not influenced by the cardinality of the dataset, indicating the robustness of our framework when the size of the dataset increases.

\section{CONCLUSIONS}

In this paper, we address the problem of deriving efficient execution plans for distributed skyline computation. A distributed skyline query can be processed by evaluating multiple constrained skyline queries on different servers. We propose a novel framework, called SkyPlan, that maps the dependencies between the queries into a graph and generates cost-aware execution plans. Our main goal is to maximize the pruning power between consecutive queries, while at the same time increase parallelism, when no significant dependencies exist among queries. Moreover, SkyPlan supports multi-objective execution plans when additional constraints - other than maximizing the pruning power - need to be enforced. Based on the derived execution plan, we propose a distributed query execution mechanism that allows continuous refinement of the execution plan during in-network query processing. In our experimental evaluation, we show that SkyPlan outperforms the state-of-the-art algorithm.

\section{REFERENCES}

[1] A. Abdalla and N. Deo. Random-tree diameter and the diameter-constrained MST. International Journal of
Computer Mathematics, 79(6):651-663, 2002.

[2] A. Amberg, W. Domschke, and S. Voß. Capacitated minimum spanning trees: algorithms using intelligent search. Combinatorial Optimization:Theory and Practice, 1:9-40, 1996

[3] W.-T. Balke, U. Güntzer, and J. X. Zheng. Efficient distributed skylining for web information systems. In Proc. of EDBT, pages 256-273, 2004.

[4] S. Börzsönyi, D. Kossmann, and K. Stocker. The skyline operator. In Proc. of ICDE, pages 421-430, 2001.

[5] L. Chen, B. Cui, H. Lu, L. Xu, and Q. Xu. iSky: Efficient and progressive skyline computing in a structured $\mathrm{P} 2 \mathrm{P}$ network. In Proc. of ICDCS, pages 160-167, 2008

[6] Y. Chu and T. Liu. On the shortest arborescence of a directed graph. Science Sinica, 14:1396-1400, 1965.

[7] B. Cui, H. Lu, Q. Xu, L. Chen, Y. Dai, and Y. Zhou. Parallel distributed processing of constrained skyline queries by filtering. In Proc. of ICDE, pages 546-555, 2008.

[8] J. Edmonds. Optimum branchings. Journal of Research of the National Bureau of Standards, 71B:233-240, 1967.

[9] D. Elias and M. Ferguson. Topological design of multipoint teleprocessing networks. IEEE Trans. on Communications, 22(11):1753-1762, 1974

[10] K. Fotiadou and E. Pitoura. BITPEER: Continuous subspace skyline computation with distributed bitmap indexes. In Proc. of DAMAP, pages 35-42, 2008.

[11] H. N. Gabow, Z. Galil, T. Spencer, and R. E. Tarjan. Efficient algorithms for finding minimum spanning trees in undirected and directed graphs. Combinatorica, 6(2):109-122, 1986.

[12] L. Gouveia. Using the Miller-Tucker-Zemlin constraints to formulate a minimal spanning tree problem with hop constraints. Computers \& Operations Research, 22(9):959-970, 1995

[13] K. Hose, C. Lemke, and K.-U. Sattler. Processing relaxed skylines in PDMS using distributed data summaries. In Proc. of CIKM, pages 425-434, 2006.

[14] Z. Huang, C. S. Jensen, H. Lu, and B. C. Ooi. Skyline queries against mobile lightweight devices in MANETs. In Proc. of ICDE, page 66, 2006.

[15] B. A. Julstrom. Greedy heuristics for the bounded diameter minimum spanning tree problem. ACM Journal of Experimental Algorithmics, 14:1.1-1.14, 2009.

[16] J. B. Rocha-Junior, A. Vlachou, C. Doulkeridis, and K. Nørvåg. AGiDS: A grid-based strategy for distributed skyline query processing. In Proc. of GLOBE, 2009.

[17] A. Vlachou, C. Doulkeridis, and Y. Kotidis. Angle-based space partitioning for efficient parallel skyline computation. In Proc. of SIGMOD, pages 227-238, 2008.

[18] A. Vlachou, C. Doulkeridis, Y. Kotidis, and M. Vazirgiannis. SKYPEER: Efficient subspace skyline computation over distributed data. In Proc. of ICDE, pages 416-425, 2007.

[19] A. Vlachou, C. Doulkeridis, Y. Kotidis, and M. Vazirgiannis. Efficient routing of subspace skyline queries over highly distributed data. Trans. on Knowledge and Data Engineering (TKDE), 22(12):1694-1708, 2010.

[20] A. Vlachou and K. Nørvåg. Bandwidth-constrained distributed skyline computation. In Proc. of MobiDE, 2009.

[21] S. Wang, B. C. Ooi, A. K. H. Tung, and L. Xu. Efficient skyline query processing on peer-to-peer networks. In Proc. of ICDE, pages 1126-1135, 2007.

[22] S. Wang, Q. H. Vu, B. C. Ooi, A. K. H. Tung, and L. Xu. Skyframe: A framework for skyline query processing in peer-to-peer systems. VLDB Journal, 18(1):345-362, 2009.

[23] P. Wu, C. Zhang, Y. Feng, B. Y. Zhao, D. Agrawal, and A. E. Abbadi. Parallelizing skyline queries for scalable distribution. In Proc. of EDBT, pages 112-130, 2006.

[24] L. Zhu, Y. Tao, and S. Zhou. Distributed skyline retrieval with low bandwidth consumption. Trans. on Knowledge and Data Engineering (TKDE), 21(3):384-400, 2009. 